10-1-2021

\title{
Frontline Health achievements in harmonizing measurement and generating evidence on community health system performance
}

Frontline Health Project

Follow this and additional works at: https://knowledgecommons.popcouncil.org/departments_sbsr-rh

Part of the Community Health and Preventive Medicine Commons, Health Policy Commons, and the Health Services Research Commons

How does access to this work benefit you? Let us know!

\section{Recommended Citation}

Frontline Health Project. 2021. "Frontline Health achievements in harmonizing measurement and generating evidence on community health system performance," Results brief. Washington, DC: Population Council. 


\section{FRONTLINE HEALTH ACHIEVEMENTS IN HARMONIZING MEASUREMENT AND GENERATING EVIDENCE ON COMMUNITY HEALTH SYSTEM PERFORMANCE}

There is growing recognition that well-supported community health workers (CHWs) are essential for effective delivery of primary health care (PHC) services, particularly in underserved, marginalized communities worldwide. As the global health community demonstrates an increasing commitment to investing in CHWs to achieve universal health coverage (UHC) in lower- and middle-income countries (LMICs), the need to effectively measure community health system performance is paramount. Embedded in the Integrating Community Health (ICH) partnership (2017-2021), with support from the Bill \& Melinda Gates Foundation (BMGF), the Population Council and Last Mile Health co-led the Frontline Health (FLH) project, a four-year research, policy, and advocacy initiative aimed at developing core metrics and $\mathrm{CHW}$ reform processes, advancing their adoption, and promoting institutionalization of robust community health policies.

This brief describes the Population Council's notable achievements and recommendations under FLH regarding CHW performance measurement, as well the top five research findings in five diverse countries: Bangladesh, Haiti, Kenya, Mali, and Uganda.

\section{DEVELOPING AND INSTITUTIONALIZING HARMONIZED METRICS TO ASSESS PERFORMANCE OF COMMUNITY HEALTH SYSTEMS}

Diligent engagement of multisectoral stakeholders promotes acceptability of measuring community health system performance.

FLH successfully engaged global and national coalitions focused on integrating data into community health programs and health management information systems. Following participatory development of the Community Health Systems Performance Measurement Framework [1], including at international conference workshops, the FLH team was well poised to socialize the framework and its related indicators and scales through existing networks such as the Health Data Collaborative, Frontline Health Worker Coalition, CHW Hub, and the National Community Health Indicators Project. FLH tested and validated the scales and tested indicators in multiple health areas (i.e., family planning (FP), maternal health, and $\mathrm{PHC}$ in four countries (Bangladesh, Haiti, Kenya, and Mali) [2,3].
Ongoing, multilevel collaboration is essential to providing effective technical leadership within key global and national partnerships.

Within the ICH partnership, FLH facilitated and co-created the development of a global research and measurement agenda through technical advisory group meetings and a co-creation workshop with partner organizations [4]. At the national level, FLH sought the expertise of and collaborated with implementing partners (Agha Khan Foundation, Zanmi Lasante, Pathfinder International, LVCT Health, Save the Children/Bangladesh, Humana HPP/DRC, Last Mile Health) to inform the framework development and research. Key donors, global development organizations, and academic partners-including the USAID, BMGF, UNICEF, the World Health Organization, World Bank (Global Financing Facility), Healthcare Information for All (HIFA), Johns Hopkins University, IntraHealth, Liverpool School of Tropical Medicine, Brigham and Women's Hospital, and the US Centers for Disease Control and Prevention-provided substantive technical inputs to inform the measurement and research agendas. In Kenya, FLH contributed to the development of the National Community Health Strategy and associated Monitoring and Evaluation Framework. 

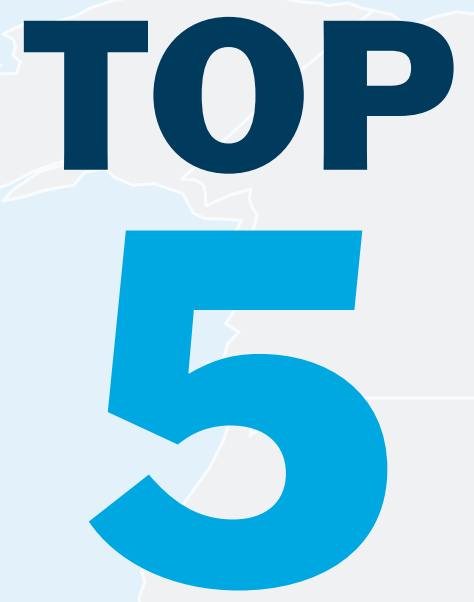

FLH

RESEARCH FINDINGS

\section{FLH \\ BY THE \\ NUMBERS}

1

Tools for assessing quality of facility-based health care can be adapted for community-based services.

Although there are many existing tools for measuring quality of health services in facilities, few have been developed and tested in community health settings. FLH successfully adapted mixedmethods strategies for assessing quality of facility-based careincluding observations of clientprovider interactions, post-service client surveys, and in-depth interviews - to measure quality of community-based postnatal care (PNC) in Kenya [5], and communitybased FP services in Bangladesh [6]. While some facility-based measures transferred well, the Method Information Index that assesses FP counseling quality, did not. Focusing on communication quality, however, is integral for quality service provision. In Kenya, communication quality was ranked high with $88 \%$ of clients reporting satisfaction in their $\mathrm{PNC}$ interactions with $\mathrm{CHWs}$, despite persisting supply challenges. Likewise in Bangladesh, clients reported moderate to high communication quality, while only $20 \%$ reported comprehensive FP counseling as measured by the Method Information Index.

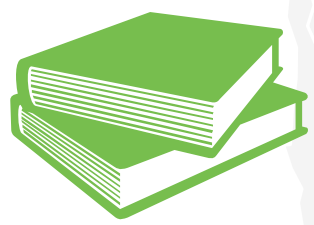

2 special issues of academic journals: Journal of Global Health and Global Health Science and Practice
2 Pragmatic financial and non-financial incentives are critical to enhancing $\mathrm{CHW}$ motivation and performance.

CHWs are vital to health systems worldwide, but they often are overworked, undermotivated, and underfunded, with insufficient supervisory or logistical support. FLH developed and validated a 22-item scale to comprehensively measure $\mathrm{CHW}$ motivation in Bangladesh and Mali [7]. To mitigate attrition and poor performance, FLH conducted discrete choice experiments in Bangladesh [8], Haiti [9], Kenya [10], and Uganda [11] to identify CHW' preferences for incentives that would improve their motivation and retention. Within both fledgling and mature $\mathrm{CHW}$ programs, CHWs favored pragmatic nonfinancial incentives such as transportation, identification badges, branded working tools (umbrellas, bags), and consistent supply of commodities and job aids. Increases in salary alone were not ranked as most important by $\mathrm{CHWs}$. The optimal incentive packages for $\mathrm{CHWs}$ are a bundle of tailored financial and non-financial offerings. Efforts to institutionalize CHWs should be accompanied by plans for sustained investments in and support for training, adequate compensation, supervision, access to working tools, and recognition of $\mathrm{CHWs}$.

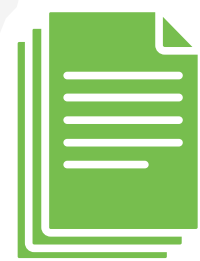

Over 15 peer-reviewed publications (https://

knowledgecommons.popcouncil. org/series_frontline_health) 
Standardized metrics for client empowerment and trust in community health systems can be applied across geographies and health specialties, with validity and reliability.

Client trust and empowerment are fundamental to an effective community health system. Clients who trust their $\mathrm{CHWs}$ have faith that these providers can meet their needs, and empowered clients possess agency to engage with the system. To fill the gap in quantitative tools to measure client trust and empowerment, FLH developed and tested the 10item Trust in CHWs Scale (Haiti, Kenya), and a 16-item Client Empowerment in Community Health Systems (CE-CHS) Scale. In all contexts, the two scales exhibited high internal consistency and reliability. These succinct scales can be incorporated into routine performance monitoring to track and improve $\mathrm{CHW}$ performance over time, as robust measures of the community health system's functioning and accountability [12,13].

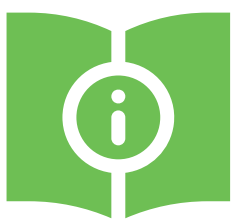

\section{Developed 20 briefs}

and guides for use by policy makers and practitioners (Access repository)
4 Digital strategies in community health can improve efficiencies in data collection and management in lowresource settings, with adequate support.

Routine data on community health systems are necessary to monitor service delivery, but data collection and management in $\mathrm{LMICs}-$ typically paper-based-often are uncoordinated, understaffed, and unreliable. In Mali, FLH conducted a pre-post mixed-method study to test the use of a District Health Information System (DHIS2) tablet-based application to improve the timeliness and completeness of community-level data submission. Compared to CHWs using paper-based data entry, those who used a tablet exhibited higher job satisfaction and productivity, as well as high confidence in ability to use the tablet. When supported with adequate connectivity, devices, and policies, the digitization of community health data collection and reporting via $\mathrm{CHWs}$ is feasible and can improve timeliness and completeness of reporting [14].

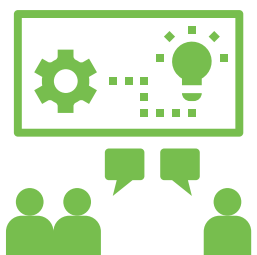

Held 8 global convenings to develop and socialize measurement and research approaches
In times of crisis or external shocks, CHWs play an essential role in sustaining health service provision and resilience within communities.

FLH investigated the effects of humanitarian crises and sociopolitical disruption on CHWs and community health systems. When these events were exacerbated by the COVID-19 pandemic, FLH proactively conducted qualitative and quantitative research to systematically assess community health responses. In Haiti, political instability, natural disasters, and disease outbreak intermittently disrupted community health system functioning. However, $\mathrm{CHWs}$ exhibit notable resilience in the face of health systems shocks, demonstrating a commitment to continuing to provide their essential services [15]. This resilience was also demonstrated in their emotional support and sustained activities during COVID-19 [16,17]. Likewise, in Bangladesh [18] and Kenya [19], $\mathrm{CHWs}$ carried on with their routine services, responding to the global pandemic by educating clients on COVID-19 prevention, symptom management, and referral. 


\section{FUTURE DIRECTIONS IN COMMUNITY HEALTH}

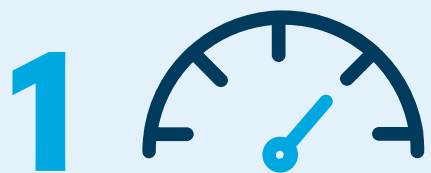

Assess integration, scale, and use of the FLH performance measures into routine community health systems monitoring, in complement with other global indicators and repositories across geographies.
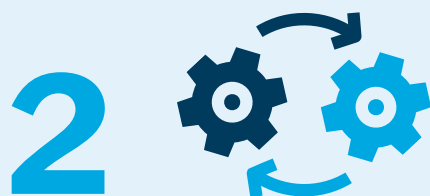

Develop and test $\mathrm{CHW}$-integrated primary health care program model acceptability and effectiveness across levels of community health systems maturity and country fragility.
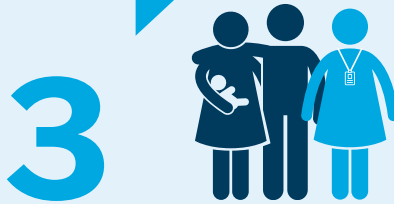

Investigate

how

supportive environments including, but not limited to financing and digital systems can enhance $\mathrm{CHW}$ performance, roles, and agency within their dynamic operational contexts.

\section{References}

1. Agarwal, S. et al. 2019a. A conceptual framework for measuring community health workforce performance within primary health care systems. Hum Resour Health. 17(2): 86. doi: 10.1186/ s12960-019-0422-0

2. Frontline Health Project. 2021. "Operationalized indicators: Integrating community health worker and client views to assess community health systems," Frontline Health Indicators Guide. Washington, DC: Population Council. doi: 10.31899/rh17.1017

3. Frontline Health Project. 2020. "Scales to measure motivation among community health workers, and trust and empowerment among their clients: A guide." Washington, DC: Population Council. doi: 10.31899/rh14.1044

4. Agarwal, S. et al. 2019b. Setting the global research agenda for community health systems: literature and consultative review. Hum Resour Health. 17: 22. doi: 10.1186/s12960-019-0362-8

5. Burnett-Zieman, B. et al. 2021. Community-based postnatal care services for women and newborns in Kenya: an opportunity to improve quality and access? J Glob Health. 11: 07006. doi: 10.7189/jogh.11.07006

6. Hossain, S. et al. 2021. Measuring quality of care at the community level using the contraceptive method information index plus and client reported experience metrics in Bangladesh. J Glob Health. 11: 07007. doi: 10.7189/jogh.11.07007.

7. Gottert, A. 2021. Development and validation of a multidimensional scale to assess community health worker motivation. J Glob Health. 11: 07008. doi: 10.7189/jogh.11.07008

8. Roy, S. et al. Beyond institutionalization: planning for sustained investments in training, supervision, and support of community health worker programs in Bangladesh. Glob Health Sci Pract. In press.

9. Frontline Health Project and Johns Hopkins Bloomberg School of Public Health. 2021. "Identifying factors that support community health worker motivation, job satisfaction, and retention in Haiti." Washington, D.C.: Population Council. doi: 10.31899/sbsr2021.1051

10. Abuya, T. et al. 2021. Incentive preferences for community health volunteers in Kenya: findings from a discrete choice experiment. BMJ Open. 11: e048059. doi:10.1136/bmjopen-2020-048059

11. Agarwal, S. et al. 2021. Understanding community health worker incentive preferences in Uganda using a discrete choice experiment. J Glob Health. 11: 07005. doi: 10.7189/jogh.11.07005

12. McClair, T. L. et al. The Client Empowerment in Community Health Systems Scale: Development and validation in three countries. J Glob Health 11: 07010. doi: 10.7189/jogh.11.07010

13. Sripad, P. et al. 2021. Measuring client trust in community health workers: A multi-country validation study. J Glob Health11: 07009. doi: 10.7189/jogh.11.07009

14. Kirk, K. et al. 2021. Introduction of digital reporting platform to integrate community-level data into health information systems is feasible and acceptable among various community health stakeholders: A mixed-methods pilot study in Mopti, Mali. J Glob Health. 11: 07003. doi: 10.7189/jogh.11.07003

15. Sripad P. et al. 2021. "Eternally restarting" or "a branch line of continuity"? Exploring consequences of external shocks on community health systems in Haiti. J Glob Health. 11: 07004. doi: 10.7189/jogh.11.07004

16. Gottert, A. et al. 2021. Extent and causes of increased domestic violence during the COVID-19 pandemic: community health worker perspectives from Kenya, Bangladesh, and Haiti. Journal of Global Health Reports. 2021;5:e2021063. doi:10.29392/001c.24944

17. Frontline Health Project and Zanmi Lasante. 2021. "Exploring community health worker roles, support, and experiences in the context of the COVID-19 pandemic in Haiti," Frontline Health Results Brief. Washington, DC: Population Council. doi: 10.31899/rh16.1020

18. Frontline Health Project. 2021. "Exploring community health worker roles, support, and experiences in the context of the COVID-19 pandemic in Bangladesh," Frontline Health Results Brief. Washington, DC: Population Council. doi: 10.31899/ rh15.1087

19. Frontline Health Project. 2021. "Exploring community health worker roles, support, and experiences in the context of the COVID-19 pandemic in Kenya," Frontline Health Results Brief. Washington, DC: Population Council. doi: 10.31899/rh14.1117

Suggested citation: Frontline Health Project. 2021. Frontline Health achievements in harmonizing measurement and generating evidence on community health system performance. Washington, D.C.: Population Council. 